\title{
Intersystem crossing in thiobases proceeds by a dark intermediate state
}

\author{
Danielle Cristina Teles-Ferreira ${ }^{1}$, Rocio Borrego-Varillas ${ }^{2}$, Lucia Ganzer $^{2}$, Barbara Elza \\ Nogueira Faria ${ }^{1}$, Cristian Manzoni ${ }^{2}$, Sandro De Silvestri ${ }^{2}$, Artur Nenov ${ }^{3}$, Irene Conti ${ }^{3}$, \\ Marco Garavelli ${ }^{*}$, Giulio Cerullo ${ }^{2 *}$, and Ana Maria de Paula ${ }^{1^{*}}$ \\ ${ }^{1}$ Departamento de Física, Universidade Federal de Minas Gerais, 31270-901 Belo Horizonte-MG, \\ Brazil \\ ${ }^{2}$ IFN-CNR, Dipartimento di Fisica, Politecnico di Milano, Piazza Leonardo da Vinci 32, I-20133 \\ Milano, Italy \\ ${ }^{3}$ Dipartimento di Chimica Industriale, Università degli Studi di Bologna, Viale del Risorgimento 4, I- \\ 40136 Bologna, Italy
}

\begin{abstract}
TU) is studied by transient absorption spectroscopy employing sub-20 fs UV-pulses and hybrid QM(CASPT2) / MM(AMBER) computations (static and dynamic), evidencing that, along the photoexcited relaxation pathway, intersystem crossing originates from a dark intermediate state.
\end{abstract}

Thiobases are DNA or RNA nucleobases where an exocyclic carbonyl oxygen is replaced by a sulfur atom [1]. Thiation induces a red-shift in the absorption spectrum of the thiobases to the $280-400 \mathrm{~nm}$ range and causes also a dramatic change in the photophysics with respect to the canonical nucleobases [2,3]: while in DNA/RNA monomers repopulation of the ground state occurs on an ultrafast timescale through a high rate of internal conversion $\mathrm{S}_{1} \rightarrow \mathrm{S}_{0}$ mediated by a conical intersection (CI), the major relaxation pathway in thiobases is via the population of triplet states via an ultrafast intersystem crossing (ISC) [4]. Thiobases have attracted much interest in the last few years due to their biological relevance and also the many possibilities of photo-therapeutic applications [1]. In spite of that, their ultrafast deactivation mechanisms are not yet fully understood. In this work we combine UV transient absorption spectroscopy featuring sub-20-fs time resolution in the UV with hybrid quantum mechanics/molecular mechanics (QM/MM) state-of-the-art computational tools to analyse the ultrafast photoinduced dynamics in 4TU. We provide the first experimental evidence that ISC occurs from a dark state of $n \pi^{*}$ character, that is intermediately populated from the bright $\pi \pi^{*}$ state excited by near-UV light [5].

Pump-probe measurements were carried out starting from a Ti:Sapphire laser producing 100 -fs pulses at $800 \mathrm{~nm}$ wavelength and $1-\mathrm{kHz}$ repetition rate. Sub-20 fs UV pump pulses (320-360 nm) were generated by frequency up-conversion of a visible NOPA with the fundamental beam (Figure 2b) [6]. The probe pulses $(250-700 \mathrm{~nm})$ were obtained by white

\footnotetext{
*Corresponding author: ana@fisica.ufmg.br, giulio.cerullo@polimi.it, marco.garavelli@unibo.it.
} 
light generation in $\mathrm{CaF}_{2}$ plate [7]. The 4TU solution was prepared in a $\mathrm{PBS}$ solution at $\mathrm{pH}$ 7.4 with concentration of $4 \mathrm{mM}$.

The QM/MM scheme couples a state-of-the-art ab-initio multireference dynamically correlated description (CASPT2) of the 4TU with an explicit classical atomistic model (AMBER force field) of the solvent [8]. A topological analysis of the populated excited states (stationary points, CIs and ISCs) is supplemented by mixed-quantum classical molecular dynamics simulations realized through a limited ensemble of 30 room temperature trajectories employing Tully's fewest switches surface hopping algorithm run for $200 \mathrm{fs}$ with a time step of $1.0 \mathrm{fs}$ at the SS-RASPT2/SA-3-RASSCF $(12,9 \mid 2,4)$ level of theory comprising all valence $\pi$-orbitals, the lone pair of the sulfur and four extra-valence virtual orbitals with up to double excitations. The state averaging covers the ground, the lowest (dark) $n \pi^{*}\left(S_{1}\right)$ and the lowest (bright) $\pi \pi^{*}$ states $\left(S_{2}\right)$, the dynamics was initiated in $\mathrm{S}_{2}$.

A $2 \mathrm{D}$ map of the differential transmission $(\Delta \mathrm{T} / \mathrm{T})$ is displayed in Figure 1a. Panel $1 \mathrm{~b}$ shows the dynamics for selected wavelengths. At early times we observe three signatures that we attribute to the bright photoexcited $\pi \pi^{*}$ state: the ground state bleaching (GSB, below $360 \mathrm{~nm}$ ) a stimulated emission (SE) signal peaking at $400 \mathrm{~nm}$ and a photo-induced absorption (PA) band above $500 \mathrm{~nm}$. The SE band displays a first ultrafast decay on the sub-100-fs timescale and subsequently a second PA band peaking at $550 \mathrm{~nm}$ grows in on a $\approx 500$-fs timescale. This band has been observed in previous studies with lower, 200-fs time resolution [4] and has been assigned to triplet-triplet absorption, thus providing a fingerprint of the ISC process. Our data clearly show that ISC does not occur directly from the photoexcited bright state but is mediated by an intermediate dark state. A global analysis of the data confirms this scenario and reveals time constants of $78 \mathrm{fs}$ and $201 \mathrm{fs}$ for the $\pi \pi^{*}$ SE decay and triplet PA formation. Furthermore, in the $2 \mathrm{D}$ map we can also clearly observe the ultrafast impulsively excited vibrational motions with two frequencies: $400 \mathrm{~cm}^{-1}$ and $680 \mathrm{~cm}^{-1}$ which are in good agreement with the calculated: a breathing mode with frequency of $457 \mathrm{~cm}^{-1}$ and in two hydrogen-out-of-plane bending modes with frequencies $697 \mathrm{~cm}^{-1}$ and $784 \mathrm{~cm}^{-1}$.
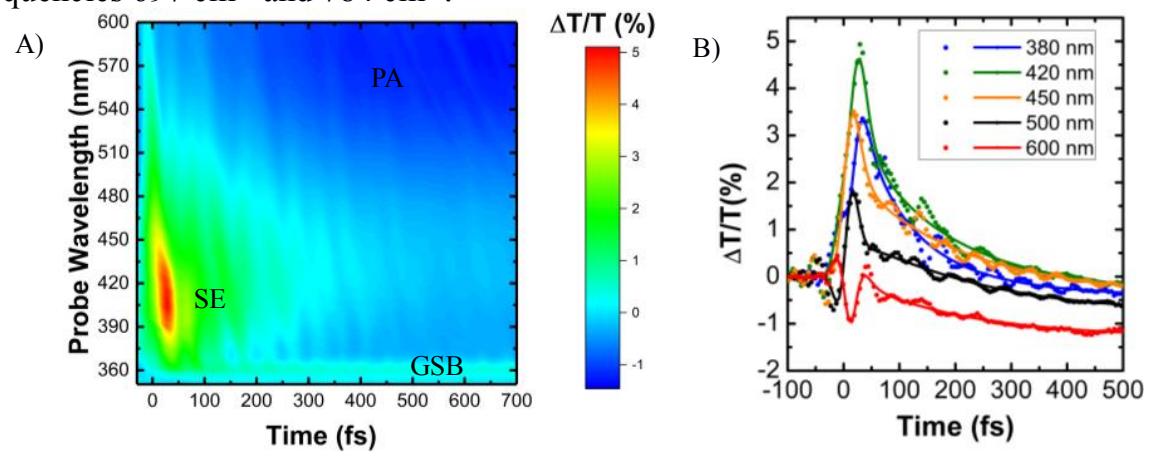

Fig 1. Transient absorption measurements: a) 2D map, b) Transient dynamics at selected wavelengths, experimental data in circles and fittings in solid lines.

By supplementing the experiments with topology analysis and molecular dynamics simulations we provide a complete picture of the photo-induced events. Figure $2 \mathrm{~b}$ shows the experimental (black) and calculated (green, blue and magenta) linear absorption spectra [9] together with the pump pulse spectrum (yellow-dashed area). The good agreement allows to assign the underlying transitions to the lowest bright $\pi \pi^{*}$ states (bands A, B and C in Figures $2 \mathrm{a}$ and $2 \mathrm{~b}$ ). The pump pulse populates the lowest $\pi \pi^{*}$ state (S2 in the Franck- 

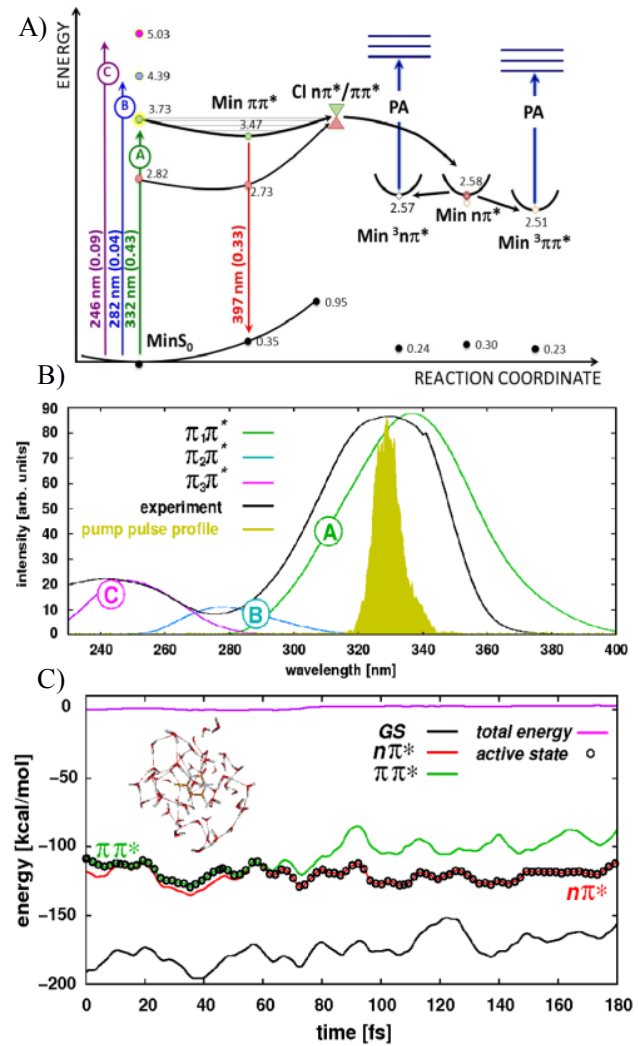

Fig 2. Computational results: a) Schematic picture of the ES deactivation processes; $b$ ) linear absorption spectrum of the lowest three bright $\pi \pi^{*}$ transitions (green, blue and magenta), experimental spectrum (black), pump pulse spectrum (yellow dashed); c) a representative molecular dynamics trajectory.

\section{References}

[1] S. Arslancan, L. Martínez-Fernández, and I. Corral, Molecules 22, 998 (2017).

[2] S. Mai, M. Pollum, L. Martínez-Fernánez, N. Dunn, P. Marquetand, I. Corral, C. E.

Crespo-Hernández and L. González, Nat. Commun 7, 13077 (2016).

[3] S. Mai, P. Marquetand, and L. González, J. Phys. Chem. A 119, 9524 (2015).

[4] M. Pollum, S. Jockusch, and C. E. Crespo-Hernández, Phys. Chem. Chem. Phys 17, 27851 (2015).

[5] R. Borrego-Varillas, D. C. Teles-Ferreira, A. Nenov, I. Conti, L. Ganzer, C. Manzoni, M. Garavelli, A. M. De Paula and G. Cerullo, submitted 2018.

[6] R. Borrego-Varillas, et al. Opt. Lett 39, 3849 (2014).

[7] R. Borrego-Varillas, L. Ganzer, G. Cerullo and C. Manzoni, Appl. Sci. 8, 2076 (2018).

[8] O. Weingart, A. Nenov, P. Altoé, I. Rivalta, J. Segarra-Martí, I. Dokukina and M.

Garavelli, J. Mol. Model., in press (2018) DOI: 10.1007/s00894-018-3769-6.

[9] A. Nenov, I. Conti, R. Borrego-Varillas, G. Cerullo and M. Garavelli, Chem. Phys. in press (2018), DOI: 10.1016/j.chemphys.2018.07.025.

DCTF, BENF and AMdeP acknowledge the CNPq, Fapemig, CAPES for financial support. 